Proc. of the 11 Int. School on Theoretical Physics Symmetry and Structural Properties of Condensed Matter, Rzeszów 2014

\title{
Density of Electron States and Relaxation Time of Intercalated Layer Crystals
}

\author{
N.K. TOVstyuK ${ }^{a, *}$ And Ye.M. SheregiY ${ }^{b}$ \\ ${ }^{a}$ National University "Lvivska Politechnika", 13, Bandera Str., L'viv, 79013, Ukraine \\ ${ }^{b}$ University of Rzeszów, Centre for Microelectronics and Nanotechnology, S. Pigonia 1, 35-959 Rzeszów, Poland \\ The density of electron states of layered crystal intercalated by guests of different nature is calculated within \\ the framework of virtual crystal considering filling of both octahedral and tetrahedral interstitial hollows. It is \\ found that the shift of the gap of density of states is caused firstly by the nature of intercalant (placing of ground \\ energy states of both intercalants) and their average concentrations. In the case when one type of intercalant gives \\ localized level in the forbidden gap and another intercalant forms the resonance one in the conductive band the \\ gap disappears. Relaxation time corresponding to the transitions from one to another band is calculated.
}

DOI: 10.12693/APhysPolA.128.225

PACS/topics: 71.20.-b, 71.55.-i, 77.22.Gm

\section{Introduction}

Intercalation of layer crystal by different guest atoms, particularly $3 d$-atoms, allows us to create new materials which can be very important for using in different aspects of applied physics [1]. The structures with alternating semiconducting and magneto-active layers can serve as materials on the base of those ones, can create the spintronics elements, particularly, as the medium structures or spin-gated transistors. Essential success in hybrid spintronics [2] is due to the intercalation of nanostructures. That is why complex studies of such structures provide additional opportunities to modify their magnetic properties in a wide range. Recently this direction is essentially enriched by theoretical studies of phase transitions considering both direct host-guest ion interaction and guest-guest interaction within the framework of pseudospin-electron model [3]. It is obvious that changes of electron spectrum of intercalated layer crystal influence to a certain degree the thermodynamics, kinetics and magnetic properties of the received intercalates.

In this paper the density of electron states of layered crystal intercalated by different guest atoms is calculated within the framework of virtual crystal considering filling of both octahedral $O$ and tetrahedral $T_{1}$ or $T_{2}$ interstitial hollows $(\mathrm{IH})$ : first ones are placed in the centrum of van der Waals gaps, $T_{1}$ or $T_{2}$ are placed closely to the monoatomic host layers nearest to the van der Waals gap (Fig. 1).

\section{Model}

Intercalant in the position $T_{1}$ or $T_{2}$ interacts with 4 nearest host atoms, in the position $O$ it interacts with 6 nearest host atoms. That is why the ground energy state of the same intercalant in tetrahedral or octahedral $\mathrm{IH}$

*corresponding author; e-mail: ntovstyuk@gmail.com

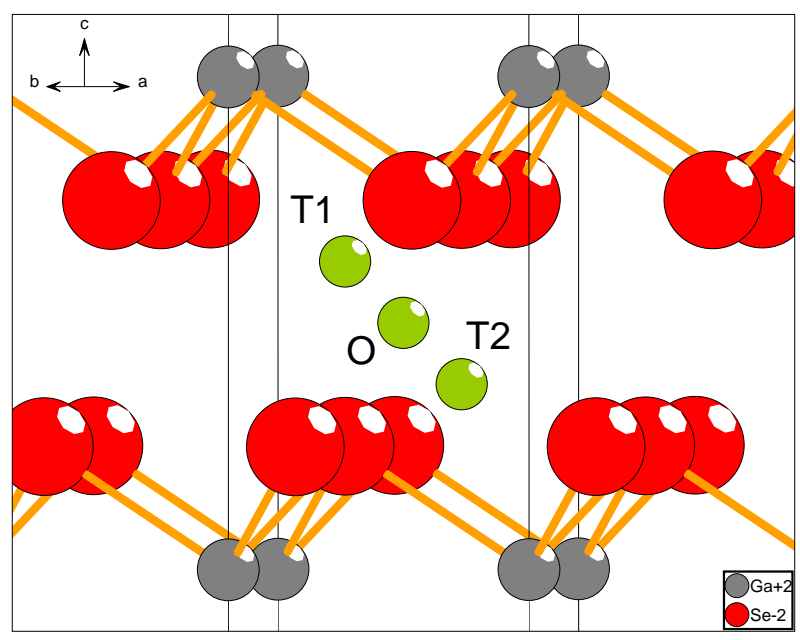

Fig. 1. Positioning of intercalant in tetrahedral $\left(T_{1}\right.$ and $\left.T_{2}\right)$ and octahedral $(O)$ IH of GaSe.

is different (electron state with energy $\varepsilon_{1}$ lies lower than another one with energy $\varepsilon_{2}$ ).

In the case when intercalant occupies positions $T_{1}$ and $T_{2}$, electret effect can occur because ordered position of intercalant can cause the appearance of intrinsic field. It consequently leads to the asymmetry of $T_{1}$ and $T_{2}$ wells. In the case of electret effect polar state in the crystal exists. Then electrons localized on the intercalant occupy IH $T_{1}$ or $T_{2}$ with different average concentrations of $p_{1}$ and $p_{2}$ and the same ground energy state $\varepsilon_{2}$.

We introduce quantum wave function $\Psi(\boldsymbol{r})$ as an expansion of wave functions of electron localized on host atom $\psi_{n}(\boldsymbol{r})$ and wave functions of electrons in the different IH $T_{1}$ and $T_{2} \varphi_{n}(\boldsymbol{r})$ and $\chi_{n}(\boldsymbol{r})$ :

$$
\begin{aligned}
& \Psi(\boldsymbol{r})=\sum_{n} c_{n} \psi_{n}(\boldsymbol{r})+\sum_{n} p_{1}(n) a_{n} \chi_{n}(\boldsymbol{r}) \\
& \quad+\sum_{n} p_{2}(n) b_{n} \varphi_{n}(\boldsymbol{r}) .
\end{aligned}
$$

A summation is carried out over all the cells with the probability $p_{1,2}(n)=1$ in the case when in the $n$-th cell 
in the position 1 or 2 intercalant exists, in the opposite case $p_{1,2}(n)=0$.

In the one-electron approximation we move to the representation of second quantization on this quantum function. The received Hamiltonian is written in the approximation of virtual crystal with constant average concentrations $p_{1}$ and $p_{2}$. Using Fourier-representation of $c_{n}, a_{n}, b_{n}$, Hamiltonian is rewritten in the quasimomentum representation as follows [4]:

$$
\begin{aligned}
H & =\sum_{\boldsymbol{k}} \varepsilon_{c}(\boldsymbol{k}) c_{\boldsymbol{k}}^{+} c_{\boldsymbol{k}}+p_{1}^{2} \sum_{\boldsymbol{k}} \varepsilon_{1}(\boldsymbol{k}) a_{\boldsymbol{k}}^{+} a_{\boldsymbol{k}} \\
& +p_{2}^{2} \sum_{\boldsymbol{k}} \varepsilon_{2}(\boldsymbol{k}) b_{\boldsymbol{k}}^{+} b_{\boldsymbol{k}}+p_{1} \sum_{\boldsymbol{k}} V_{1}(\boldsymbol{k}) c_{\boldsymbol{k}}^{+} a_{\boldsymbol{k}} \\
& +p_{2} \sum_{\boldsymbol{k}} V_{2}(\boldsymbol{k}) c_{\boldsymbol{k}}^{+} b_{\boldsymbol{k}}+p_{1} p_{2} \sum_{\boldsymbol{k}} V_{12}(\boldsymbol{k}) a_{\boldsymbol{k}}^{+} b_{\boldsymbol{k}}+\text { H.c. }
\end{aligned}
$$

where $\varepsilon_{c}(\boldsymbol{k})$ coincides with dispersion law of the conduction band of layer crystal

$$
\varepsilon_{c}(\boldsymbol{k})=\alpha\left(k_{x}^{2}+k_{y}^{2}\right)+t\left(1-\cos \left(k_{z} d_{z}\right)\right),
$$

where $\alpha$ is effective mass of electron within the layer plane, $t$ - electron mixing among the nearest layers along the anisotropy axis. Here and below $\hbar=1$. Two additional bands, created by electrons localized on the intercalant in the different $\mathrm{IH}$ are described as follows:

$$
\begin{aligned}
& \varepsilon_{1}(\boldsymbol{k})=\alpha_{1}\left(k_{x}^{2}+k_{y}^{2}\right)+t_{1}\left(1-\cos \left(k_{z} d_{z}\right)\right)+\varepsilon_{1}^{0} / p_{1}, \\
& \varepsilon_{2}(\boldsymbol{k})=\alpha_{2}\left(k_{x}^{2}+k_{y}^{2}\right)+t_{2}\left(1-\cos \left(k_{z} d_{z}\right)\right)+\varepsilon_{2}^{0} / p_{2} .
\end{aligned}
$$

Two time retarded Green functions are considered for $\left\langle\left\langle c_{\boldsymbol{k}} \mid c_{\boldsymbol{k}}^{+}\right\rangle\right\rangle$layer lattice and for intercalant $\left\langle\left\langle a_{\boldsymbol{k}} \mid a_{\boldsymbol{k}}^{+}\right\rangle\right\rangle$ $\left\langle\left\langle b_{\boldsymbol{k}} \mid b_{\boldsymbol{k}}^{+}\right\rangle\right\rangle$. Motion equation for $\left\langle\left\langle c_{\boldsymbol{k}} \mid c_{\boldsymbol{k}}^{+}\right\rangle\right\rangle,\left\langle\left\langle a_{\boldsymbol{k}} \mid a_{\boldsymbol{k}}^{+}\right\rangle\right\rangle$and $\left\langle\left\langle b_{\boldsymbol{k}} \mid b_{\boldsymbol{k}}^{+}\right\rangle\right\rangle$is written.

These results allow us to receive density of electron states of the intercalated layer crystal (Fig. 2).

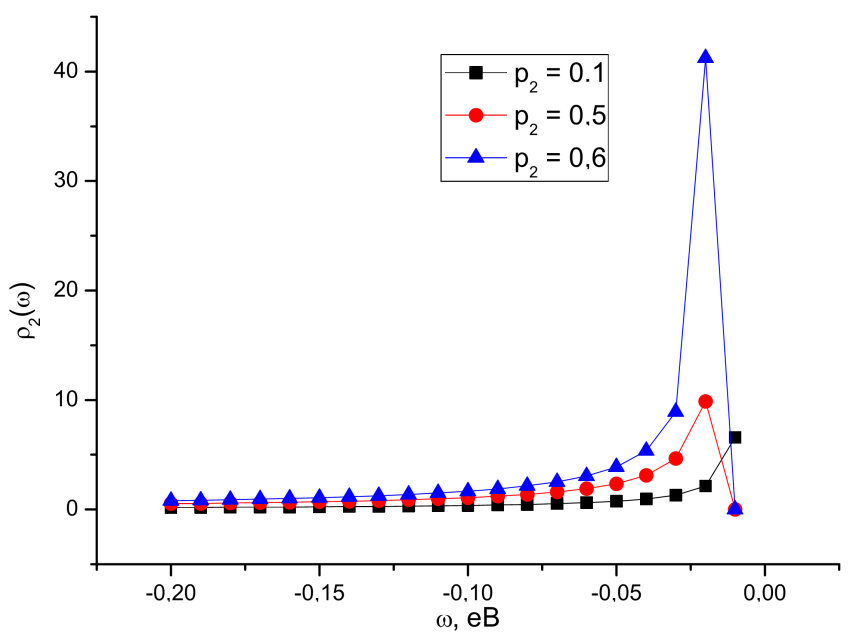

Fig. 2. Density of electron states of intercalated layer crystal with intercalant in $O$ and $T \mathrm{IH}$ and chosen values of ground energies $\varepsilon_{1}=-0.1 \mathrm{eV}, \varepsilon_{2}=-0.02 \mathrm{eV}$.

\section{Results and discussion}

In the case of occupying $O$ and $T$ IH it is found that additional gap appears in the forbidden gap (Fig. 2); its shift is caused firstly by the nature of intercalant (positioning of ground energy states of electron localized on the intercalant in $O$ and $T \mathrm{IH}$ ) and their average concentrations. In the case when one type of intercalant gives localized level in the forbidden gap and another intercalant forms the resonance one in the conductive band the gap disappears. It is shown that if electron mixing between three zones is absent, intercalant system behaves independently of the layer crystal system. In the case when electret effect appears we consider positioning of the same intercalant in two wells $T_{1}$ and $T_{2}$ of different depth and thus only concentrations $p_{1}$ and $p_{2}$ are different. Our calculations showed that if $p_{1}=0.98$ and $p_{2}$ changes from 0.2 up to 0.7 (Fig. 3) the width of additional gap changes in monotonic way. The shift of the additional gap depends strongly on the value of the intercalant ground energy state $\left(\varepsilon_{2}\right)$, i.e. where does it lies below or above the bottom of the conduction band.

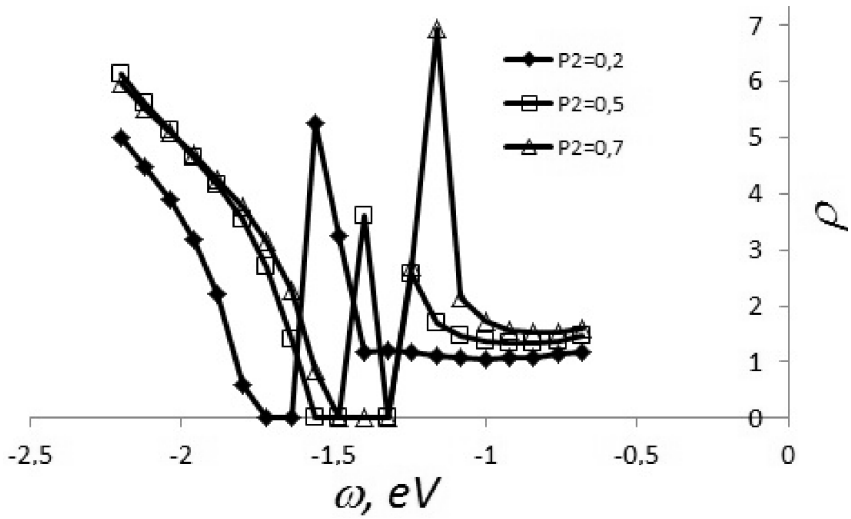

Fig. 3. Density of electron states of intercalated layer crystal with intercalant in $T_{1}$ and $T_{2} \mathrm{IH}, p_{1}=0.98$, different $p_{2}$ and corresponded ground energies $\varepsilon_{1}=\varepsilon_{2}=$ $-0.1 \mathrm{eV}$.

Relaxation time is calculated in the both cases. We found that in the case of electret effect it is an order higher than in the case of occupying symmetry different IH.

\section{Conclusions}

Density of electron states of the intercalated layer crystal considering different type of intercalant (atoms, ions, molecules) and possibility of occupying different interstitial hollows are studied. It is found that the shift of the additional gap in the density of electron states primarily depends on the intercalant nature, i.e. mutual positioning of $\varepsilon_{1}$ and $\varepsilon_{2}$ and their average concentrations $p_{1}$ and $p_{2}$. Additional gap can disappear if electron localized on the one type of intercalant gives resonance level in the conduction band $\left(\varepsilon_{1}>0\right)$, and an electron localized 
in the another well gives localized level $\left(\varepsilon_{2}<0\right)$. The proposed model allows to describe electron energy structure of layer crystal with intercalant component which possesses large dipole moment. Ordered distribution of dipoles in the van der Waals gap in respect of the sandwiches of layer crystal causes the appearance of additional intrinsic field which shifts energy levels of intercalant. In the case of random dipole distribution such field disappears and thus the value of energy shift disappears. Considering polar state of the crystal we assume that the same type of intercalant appears in two wells formed by $T_{1}$ and $T_{2} \mathrm{IH}$. The depth of these wells is different. Thus only concentrations $p_{1}$ and $p_{2}$ are different. Our calculations showed that the width of additional gap in the density of electron states changes in monotonic way depending on the change of average concentration in one of $T \mathrm{IH}$.

\section{References}

[1] M. Elhabiri, A.-M. Abrecht-Gary, Coord. Chem. Rev. 252, 1079 (2008).

[2] B.P. Zakharchenya, Phys.-Usp. 175, 625 (2005).

[3] I.V. Stasyuk, Yu.I. Dublenych, Phys. Rev. B 72, 224209 (2005).

[4] N.K. Tovstyuk, Visnyk Lviv Univ. Ser. Phys. 48, 109 (2013). 